\title{
BIO-ENGINEERING ASPECTS
}

\author{
Chairman: Professor R. M. Kenedi
}

\section{Introduction}

\author{
R. M. KENEDI \\ Bio-engineering Unit, University of Strathclyde, Glasgow
}

LADIES AND GENTLEMEN, may I commence by welcoming you all very cordially on behalf of the organizers of the Symposium. Particularly we extend a welcome to our visitors from Canada, Holland, Sweden, Switzerland and U.S.A. Also I have a telegram from Mr Dickson, Chairman of the Hospital Management Committee, Wigan and Leigh Group of Hospitals, who conveys his felicitations for the success of the conference.

Patient monitoring is normally used in a clinical context to assist in the provision of concentrated care and so improve the survival of patients subjected to or affected by life-threatening conditions. A further development of these facilities, particularly in relation to intensive care, has in part been generated by the limited availability of highly specialized nursing personnel. Relevant publications both in this country and abroad suggest that there is a critical shortage of professional nurses trained particularly in the care of the seriously ill. Interestingly this area is labour intensive and it is estimated that the average daily nursing requirement for a normal patient is about $5 \mathrm{hr}$ while that for a critically ill patient is between $9-13 \mathrm{hr}$. Correspondingly the cost is higher and therefore there is an incentive to introduce technological aids. While therefore it can be said that monitoring systems have been developed under pressure of lack of availability of labour, I think it is also true to say that, with the advance of technology, technological aids have become part of the clinical picture and their use is becoming part of the basic skills of nursing. Being a technologist one does, in off moments, admit that technology at times tends to run away with itself and this I think is happening in patient monitoring also. There are systems now where something like eleven primary measurements are monitored giving something like twenty-six derived variables on-line, backed up by another eleven off-line parameters obtained manually. It is not surprising, therefore, that the physician who is charged with the diagnostic and therapeutic care of the patient gets a bit confused and some of these more sophisticated monitoring systems have to include a primary diagnosis facility if for no other reason than to draw attention of the physician as to what is significant and what he can safely disregard.

In this context $I$ think there are two major areas within monitoring systems that could be further developed. One the area of sensors: present development in this field is very largely oriented to the evaluation of normal physiological variants. In fact what is probably required is the assessment of the patho-physiological parameters. The second area is one that has intrigued me ever since I came into this field as an engineer and began watching how medically trained people work. Diagnosis entirely from a layman's view is a sort of continual toing and froing between the clinician and the patient, as the clinician selectively recalls his relevant past experience in sequential response to the patient's description of his symptoms. This gradually builds up the diagnostic picture, and quite frankly it is just not conceivable that this, certainly in the foreseeable future, could be replaced in any way by automated systems. Against this, the system, provided it is genuinely designed to assist the physician in making a decision can accelerate and objectivize this decision very effectively. Thus the clinician is the centre of the system, effectively integrated with it but by no stretch of imagination replaced by it. 